\title{
Personal experience on the use of PMIC
}

\author{
M D’Andrea ${ }^{*}$, L Scalzo $^{1}$, L Leo $^{2}$, D De Buono ${ }^{3}$, M locco $^{1}$ \\ From de Senectute: Age and Health Forum \\ Catanzaro, Italy. 5-7 December 2009
}

\section{Background}

The Calabria group of the PMIC reports its experience, on the application of the protocol of minimal appraisal in some rehabilitative structures that represent the possible different settings for strokes in the Calabria Region. The total data will be exposed. The number of the collate cards exceeds 450 cases, thus constituting a meaningful collection of regional data that could be used in order to photograph the state of the patient with a stroke in Calabria and in order to program more and more appropriate rehabilitative intervention and settings, in order to rationalize the use of the employed resources.

\footnotetext{
Author details

${ }^{1}$ Cattedra di Medicina Fisica e Riabilitazione, Università degli Studi "Magna Græcia", Catanzaro, Italy. ' U.O. di Medicina Fisica e Riabilitazione, Azienda Ospedaliera "Dell'Annunziata", Cosenza, Italy. ${ }^{3}$ Casa di cura "M. Misasi", Reparto Riabilitazione, Cosenza, Italy.
}

Published: 19 May 2010

\section{References}

1. SPREAD IV edizione: linee guida italiane di prevenzione e trattamento dell'ictus cerebrale. Stesura del 15 marzo 2005.

2. Linn FH, Rinkel GJ, Algra A, van GijnJ: Incidence of subarachnoid hemorrhage: role of region, year, and rate of computed tomography: a meta-analysis. Stroke 1996, 27:625-629.

3. Hop JW, Rinkel GJ, Algra A, van Gijn J: Quality of life in patients and partners after aneurismal subarachnoid hemorrhage. Stroke 1998, 29:798-804.

4. Villella S, Catanzaro T, Colao A M, De Buono D, Leo L, Longo C, Mileto A, Scalzo L, locco M: Esperienza del gruppo calabrese sull'utilizzo del protocollo di valutazione di minima nelle persone con ictus cerebrale (PMIC). Eur Med Phys 2007, 43(suppl. 1 to no. 3):1-3.

5. Nocella A, Sciarrini f, Orecchini g, Todeschini e, schifini $m$ f, Braconi a, Zampolini $m$, Massucci m: Il protocollo di minima di valutazione dell'ictus cerebrale (PMIC) in Umbria: I'esperienza di due centri di riabilitazione. Abstract Book VIII Congresso Nazionale SIRN 231-233.

6. Piazzini D B, Ferrara P E, Maggi L, Frasca G, Aprile I, Rabini A, Specchia A, Bertolini C: Application of "Protocollo di Minima per I'Ictus" (PMIC Minimal Protocol for Stroke) for inpatient rehabilitation setting. European

\footnotetext{
${ }^{1}$ Cattedra di Medicina Fisica e Riabilitazione, Università degli Studi "Magna
} Græcia", Catanzaro, Italy

Journal of Physical and Rehabilitation Medicine (Europa Medicophysica) 2008, 44(3).

7. Lenti G, Agosti M, Massucci M, Zampolini M, Paolucci S, Franceschini M: Developing a minimum data set for stroke patients assessment: the "Protocollo di Minima per l'Ictus" (PMIC) as a starting point towards an Italian stroke registry. Eur J Phys Rehabil Med 2008, 44(3):263-9.

doi:10.1186/1471-2318-10-S1-L59

Cite this article as: D'Andrea et al:: Personal experience on the use of PMIC. BMC Geriatrics 2010 10(Suppl 1):L59.
Submit your next manuscript to BioMed Central and take full advantage of:

- Convenient online submission

- Thorough peer review

- No space constraints or color figure charges

- Immediate publication on acceptance

- Inclusion in PubMed, CAS, Scopus and Google Scholar

- Research which is freely available for redistribution

Submit your manuscript at www.biomedcentral.com/submit
C Biomed Central 